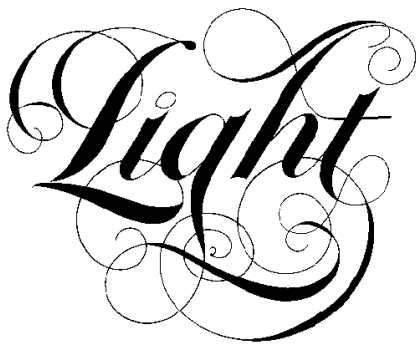

\title{
Efficient Superfluorescent Light Sources with Broad Bandwidth
}

\author{
R.Paschotta, J.Nilsson, A.C.Tropper and D.C.Hanna
}

\begin{abstract}
We demonstrate various efficient broad-band light sources at $\sim 1 \mu \mathrm{m}$ wavelength with a $3 \mathrm{~dB}$ bandwidth of up to $65 \mathrm{~nm}$ at $108 \mathrm{~mW}$ output power, based on rare-eartb doped silica fibers and a simple adjustable spectral filter.
\end{abstract}

\section{Introduction}

Low-coherence light sources with high-spectral power density over a broad wavelength range in a single spatial mode are needed for a number of applications such as optical gyroscopes, for the characterization of optical devices, and for interferometric techniques as used, e.g., in low-coherence reflectometry or in medical imaging of soft tissues. Such light sources can be made with rare-earth doped single-mode fibers, generating amplified spontaneous emission (ASE). Both neodymium-doped [1] and erbium-doped [2] fibers have been used to produce ASE output powers of hundreds of milliwatts, however with a FWHM bandwidth below 5nm, while more bandwidth has been achieved at lower output powers, typically up to 30nm for output powers well below 100mW [3], [4]. This paper describes a way to achieve an even much larger bandwidth of up to $65 \mathrm{~nm}$ at an output power of more than $100 \mathrm{~mW}$.

\section{Application of spectral filtering}

The achieved bandwidth of emission of a superfluorescent source is usually significantly smaller than the range in which spontaneous emission and amplification is observed, because the spectral power density generated by ASE depends critically on the wavelength-dependent emission and absorption cross sections, particularly when the device has high gain as required for high-output power and high-conversion efficiency. The general route to achieving emission with broader bandwidth is spectral filtering, which need not decrease the power efficiency if applied to a low-power seed before high-power amplification rather than to the output. One possibility is to use a two-stage device where the low-power output of the first stage is first spectrally filtered and then amplified to high power in the second stage; such a filter would be designed to decrease the spectral power density of the seed (the input of the second stage) in the wavelength regions of high gain. High-power efficiency can be achieved in a simple configuration by using the forward ASE of a single amplifier to generate the seed for amplification in the backward direction. We have taken this route in our experiments, similar to the approach taken in [4] where, however, no filtering was applied.

\section{The experiments}

The spectral filter used for a broad-band superfluorescent source would ideally be realized in all-fiber technology in order to obtain maximum stability of the device. The most attractive solution for commercial devices will probably be the use of UV-written large period fiber gratings which generate optical loss by coupling the propagating mode to cladding modes [5]; care will be needed to produce such gratings with sufficient accuracy, and such a filter is of course not adjustable once produced. For the work presented in this paper we used a freely adjustable 
spectral filter of a rather crude construction using bulk optics, but which can easily be made and adjusted to maximize the bandwidth of an ASE source. While the use of bulk optics is not ideal for a commercial device, it is acceptable for a research tool where the advantages of easy construction and adjustability are often more important. Our filter consists of a pair of bulk gratings to separate the different wavelengths spatially, and a simple spatial modulator, realized in the form of an array of fine screws of $1.5 \mathrm{~mm}$ diameter, projecting from threaded holes spaced by $4 \mathrm{~mm}$ (center-to-center) in a $12 \mathrm{~cm}$-long aluminum bar. This array is mounted above the beam so that each screw can attenuate a particular wavelength range of a few nm bandwidth when positioned so as to partially obstruct the beam. The wavelength ranges can be made to overlap by mounting the array at an appropriate angle to the beam. A good performance was achieved when the extreme wavelength components were separated by about $2.5 \mathrm{~cm}$ and the screw array was angled so that it could just attenuate the full wavelength range.

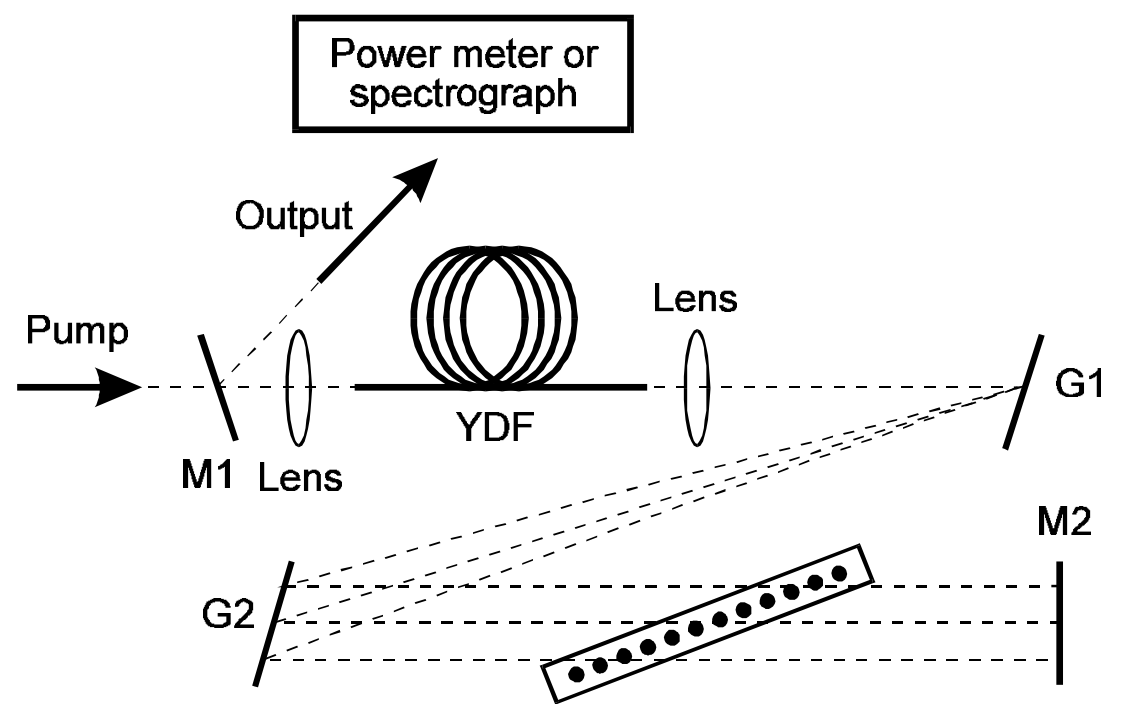

Fig. 1. The experimental setup. M1: Dichroic mirror. YDF: Ytterbium-doped fiber. G1, G2: Gratings with 600 lines/mm. M2: high reflector. Between G2 and M2: Array of screws as attenuator.

Fig. 1 shows the first experimental set-up. The 980-nm pump beam from a Ti:sapphire laser was launched through an optical isolator, the dichroic mirror M1 and the aspheric lens Ll into a $4 \mathrm{~m}$-long ytterbium-doped germano-silicate fiber with a numerical aperture of 0.21 , a cutoff wavelength of $930 \mathrm{~nm}$, and an ytterbium-concentration of 2300ppm by weight. We have chosen to use ytterbium-doped fiber mainly because of its very broad amplification bandwidth although the described method could be applied as well with other dopants, e.g., with erbium for the wavelength region around $1550 \mathrm{~nm}$. Both fiber ends were angled at roughly $10^{\circ}$ in order to suppress reflections. The forward ASE appearing at the output end of the fiber was collimated with an achromatic microscope objective and spatially dispersed by the gratings G1 and G2. The attenuator was placed just after G2, and finally the beam was reflected back by the broad-band silvered mirror M2. The output was separated from the pump beam by mirror M1 which had high transmission for the $980 \mathrm{~nm}$ pump beam and more than $99 \%$ reflectivity for $1020-1230 \mathrm{~nm}$. The spectral shape of the output was monitored with a fast grating spectrometer (based on a silicon diode array, calibrated for uniform spectral response); alternatively the total power could be measured with a thermal power meter. 
The alignment procedure for obtaining maximum bandwidth of the output can be performed within a few minutes. First, the feedback is aligned without the attenuator. The beam diameter should be at least $1 \mathrm{~mm}$ at the position of the attenuator in order to achieve a smooth spectral response. The attenuator is first mounted with all screws fully down into the beam (maximum attenuation); in this situation the output consists only of single-pass backward ASE. Finally, the screws are successively screwed upwards so as to enhance the power in all the weaker wavelength channels to roughly the same power as in the strongest channel (at $1035 \mathrm{~nm}$ ). Some fine tuning allows one to achieve quite a flat spectrum with excursions of only $\sim 0.5 \mathrm{~dB}$ (peak-to-peak) in a broad wavelength range.

The solid curve in Fig. 2 shows the output spectrum obtained for $295 \mathrm{~mW}$ of incident input power (measured at the lens L1), which corresponds to roughly $150 \mathrm{~mW}$ of launched power. The total output power was $70 \mathrm{~mW}$. The full-width at half-maximum (FWHM) bandwidth is $39 \mathrm{~nm}$. Note that the vertical scale is linear; on a logarithmic scale with, e.g., a 30dB range the residual wiggles on the spectrum would be hardly visible. Despite the simple design of the attenuator the stability of the spectral shape was good; over 5 min of operation we typically observed a drift

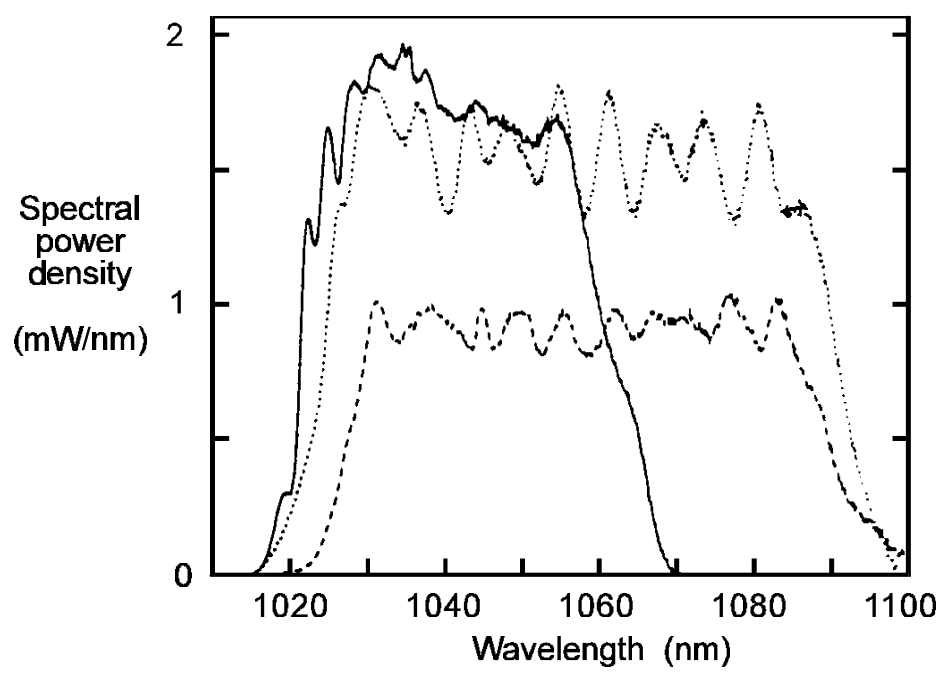

Fig. 2. Output spectra of various ASE sources. Solid curve: with $980 \mathrm{~nm}$ pump, $70 \mathrm{~mW}$ output power. Dashed curve: with $1010 \mathrm{~nm}$ pump, 62mW output. Dotted curve: with $980 \mathrm{~nm}$ pump and an additional Nd-doped fiber, pumped at $815 \mathrm{~nm}, 108 \mathrm{~mW}$ output power.

of the total output power by $10 \%$ (due to changes in the Ti:sapphire output power), but no change in the spectral shape.

In order to extend the bandwidth even further, we changed the pump wavelength to $1010 \mathrm{~nm}$, generating a lower $\mathrm{Yb}^{3+}$ inversion density and broader gain spectrum. and used a longer fiber length $(15 \mathrm{~m})$ because of the lower pump absorption. In this way we achieved a very smooth and broad spectrum (dashed curve in Fig. 2) with $62 \mathrm{~nm}$ FWHM at $62 \mathrm{~mW}$ output power, using $366 \mathrm{~mW}$ of incident pump power; the power efficiency was lower than before because the lower pump absorption made this configuration more sensitive to background loss of the fiber.

Another way to extend the emission bandwidth to longer wavelengths is to increase the gain in this region by addition of a neodymium-doped fiber. Modifying the setup as described above, we fusion-spliced a 3m-long Nd-doped single-mode silica fiber (codoped with some germanium and 
aluminum) to the 4m-long $\mathrm{Yb}$-doped fiber (the opposite end to the pump input end) and used a wavelength selective fiber coupler to inject pump light from a single-stripe laser diode with $100 \mathrm{~mW}$ at $815 \mathrm{~nm}$. Only about $30 \mathrm{~mW}$ of $815 \mathrm{~nm}$ pump power could be launched into the Nd-doped fiber; this was not sufficient to generate more than a few milliwatts of ASE in the $\mathrm{Nd}$-doped fiber alone, but in combination with the fluorescence and gain of the Yb-doped fiber this was enough to generate a broad peak around $1060 \mathrm{~nm}$ in the single-pass spectrum (i.e., with the attenuator fully blocked). By adjusting the attenuator we then achieved the bandwidth of $65 \mathrm{~nm}$ at $108 \mathrm{~mW}$ of output power, shown as the dotted curve in Fig. 2. Compared to pumping at $1010 \mathrm{~nm}$, the advantages are the higher output power, the slightly broader bandwidth, and the availability of powerful diode lasers around 815 and $980 \mathrm{~nm}$.

The double-pass scheme has the disadvantage of being sensitive to small reflections of the output into the source. Indeed we observed that good adjustment of the optical isolator was necessary, and spurious lasing spikes in the spectrum could not be eliminated when the pump power was set higher than $\sim 350 \mathrm{~mW}$ (incident at L1). However, if necessary the demonstrated principle could be used in a two-stage single- pass scheme with the spectral filter between the stages. This should allow a higher output power and a slight further increase in output bandwidth because the gain of the power amplifier could be smaller. The Ti:sapphire pump laser of our set-up could be replaced by a diode laser to achieve a high wall-plug efficiency and higher output power stability.

\section{Conclusion}

A large emission bandwidth of a superfluorescent source can be achieved together with high output power and high efficiency by applying a double-pass (or two-stage) scheme and a suitable spectral filter. We have made an adjustable spectral filter of simple design and applied it to broaden the spectra of various ASE sources. A FWHM bandwidth of up to $65 \mathrm{~nm}$ at $108 \mathrm{~mW}$ of output power was achieved.

\section{Acknowledgment}

The authors thank L.Dong and D.J.Richardson for useful discussions.

\section{References}

1. J.D.Minelly, P.R.Morkel, K.P.Jedrzejewski, E.R.Taylor, J.Wang and D.N.Payne, "Nd ${ }^{3+}$-doped singlemode fiber superfluorescent source with $320 \mathrm{~mW}$ output power," Electron. Lett., vol. 29, pp. 1613-1614. 1993.

2. S.Gray, J.D.Minelly, A.B.Grudinin and J.E.Caplen, "Superfluorescent Er/Yb single-mode fiber source with 1 Watt output power," in CLEO'97 Tech. Dig. Baltimore, MD, pp. 82-83, 1994, paper CTUG7.

3. P.W.Wysocki, M.J.F.Digonnet, B.Y.Kim and H.J.Shaw, "Characteristics of erbium-doped superfluorescent fiber sources for interferometric sensor applications," J. Lightwave Tech., vol. 12, pp. 550-567, 1994

4. L.A.Wang and C.D.Chen, Electron. Lett., vol. 32, pp. 1815-1817. 1996.

5. A.M.Vengsarkar, P.J.Lemaire, J.B.Judkins, V.Bhatia, T.Erdogan and J.E.Sipe, "Long-period fiber gratings as band-rejection filters," J.Lightwave Technol., vol. 14, pp. 58-65, 1996. 